\title{
Business Process Failure Prediction: a case study
}

\author{
Pedro O. T. Mello ${ }^{1}$, Kate Revoredo², Flávia Santoro ${ }^{3}$ \\ 1 Federal University of the State of Rio de Janeiro, Rio de Janeiro, Brazil \\ pedro.mello@uniriotec.br \\ 2 Federal University of Rio de Janeiro, Rio de Janeiro, Brazil \\ katerevoredo@ppgi.ufrj.br \\ 3 State University of Rio de Janeiro, Rio de Janeiro, Brazil \\ flavia@ime.uerj.br
}

\begin{abstract}
Business process monitoring aims at maintaining the reliability of process executions. However, the dynamic nature of business processes hinders a proactive scenario in which risk mitigation actions can occur before the facts that put the process at risk. Thus, some premises are necessary such as the identification of situations and patterns in historical data of the processes execution in order to characterize what determined the failures. In this paper, we address the problem of how to identify and detect patterns of behaviors that can lead the processes to a failure situation. As a solution, a combination of well-established techniques from Data and Process Mining fields are applied in a case study of an incident management process. The results obtained open possibilities to a proactive scenario.
\end{abstract}

Categories and Subject Descriptors: H.2.8 [Database Management]: Database Applications; I.2.6 [Artificial Intelligence]: Learning

Keywords: Data Mining, Business Process, Process Monitoring, Failure Prediction

\section{INTRODUÇÃO}

Processos de negócio visam o alcance dos objetivos estratégicos e operacionais de um ambiente organizacional por meio de atividades coordenadas [Van Der Aalst 2013]. A dificuldade no gerenciamento de processos está associada a dois fatores: a sua natureza dinâmica; e o desconhecimento por parte de analistas e gestores de determinadas situações que exercem influência no seu desempenho e fluxo de atividades [Hompes et al. 2017]. Esses fatores aumentam a complexidade de estratégias proativas. Em um cenário reativo, decisões e estratégias de mitigação de riscos acontecem somente após a ocorrência de eventos ou atividades indesejadas. Por outro lado, um cenário proativo possibilita a tomada de decisão antes da ocorrência de eventos ou atividades indesejadas. Assim, uma abordagem proativa seria possível a partir de estimativas de uma realidade empírica, sendo portanto considerada preditiva. A Gestão de Processos pode delimitar essas estimativas a partir dos indicadores de desempenho do processo. Em geral, indicadores são definidos como métricas que podem identificar problemas de desempenho[del Río-Ortega et al. 2017].

Este artigo destaca atividades de monitoramento que visam medir o desempenho de processos sem a necessidade de construir ou utilizar um modelo de processo [van der Aalst 2016]. Esta premissa aponta para o desafio inerente ao fornecimento de apoio operacional a atividades de detecção, predição e recomendação, tratado como um dos onze desafios ainda em abertos da área de Mineração de Processos. A Mineração de Processos tem como objetivos descobrir, monitorar e aprimorar processos de negócio por meio da extração de conhecimento a partir de dados; portanto integra técnicas e conceitos de Gerenciamento de Processos de Negócio, Mineração de Dados e Aprendizado

Copyright@2019 Permission to copy without fee all or part of the material printed in KDMiLe is granted provided that the copies are not made or distributed for commercial advantage, and that notice is given that copying is by permission of the Sociedade Brasileira de Computação. 
de Máquina [van der Aalst 2016].

Diversas técnicas de mineração de processos têm sido propostas para aprimorar o gerenciamento de processos, sejam em cenários que visam à melhoria de processos de negócio ou em cenários de diagnóstico do processos [Rudzajs and Kirikova 2018; Weske et al. 2018]. Cenários de diagnóstico tem por objetivo a busca por explicações da existência de determinadas características das instâncias do processo e, em alguns casos, usar o conhecimento adquirido para estimar uma realidade empírica e criar abordagens preditivas.

O objetivo deste artigo é apresentar um método, que combina diferentes técnicas de mineração de dados para a predição de fracassos em processos de negócio. Neste sentido, o fracasso é determinado por meio de uma situação que apresenta risco ao negócio. Essa situação é determinada pelo acordo a nível de serviço (SLA), que é um compromisso assumido por um prestador de serviços perante um cliente [del Río-Ortega et al. 2015]. O não cumprimento do SLA apresenta riscos ao negócio, podendo ocasionar em prejuízos financeiros para uma organização ${ }^{1}$. O método proposto foi avaliado em um estudo de caso com dados reais do departamento de tecnologia da informação de uma organização brasileira. Desta forma, a seguinte questão de pesquisa foi formulada: [QP] Como combinar e aplicar as técnicas de Mineração de Dados e Mineração de Processos para a predição de fracassos no domínio de gerenciamento de incidentes? A principal contribuição é a definição de estratégias de mitigação de riscos durante a execução de processos.

O artigo está estruturado da seguinte forma: a Seção 2 descreve o cenário, o aparato experimental e a construção do modelo de predição; a Seção 3 discute os resultados; a Seção 4 apresenta uma breve síntese de trabalhos que antecederam a esta pesquisa; a Seção 5 apresenta as principais conclusões e trabalhos futuros.

\section{PROJETO EXPERIMENTAL}

Esta seção apresenta os passos propostos para a modelagem da predição das situações de fracassos. Esses passos envolvem a combinação de diferentes técnicas oriundas da Mineração de Dados e Mineração de Processos e pré-processamento. O método proposto é apresentado na Figura 1.

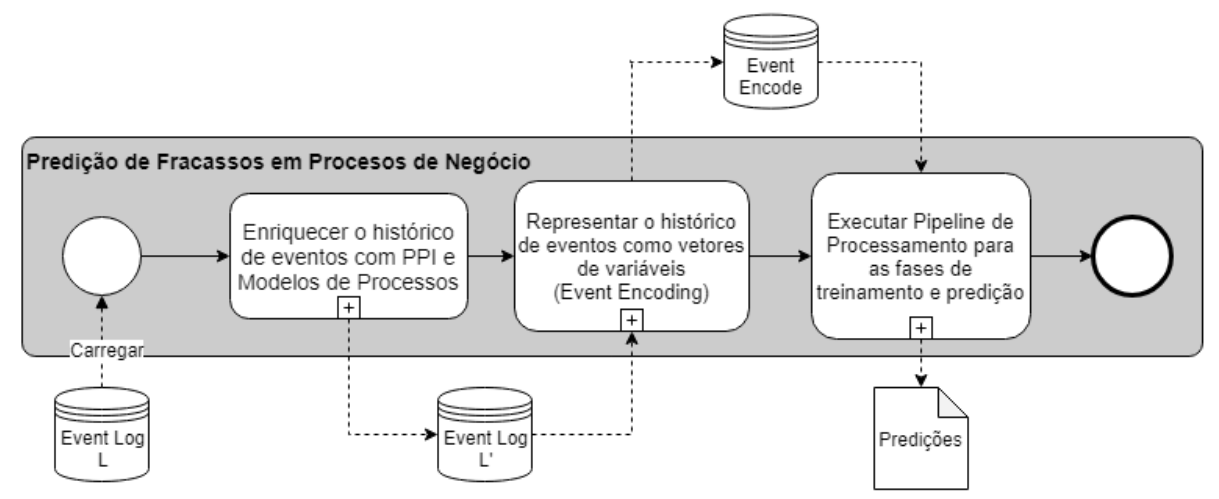

Fig. 1: Etapas do método para a predição de fracassos em processos de negócio.

As próximas seções apresentam a aplicação de método no cenário do estudo de caso.

\footnotetext{
${ }^{1}$ Em geral, a penalidade aplicada são multas que podem ser arcadas por meio de pagamentos a empresa lesada pelo não cumprimento do serviço ou redução do valor do contrato.
} 


\subsection{Histórico de Eventos}

Os dados de entrada para a aplicação de qualquer técnica de Mineração de Processos é um histórico ou log de eventos (Event Log). Esse histórico é uma sequência de eventos originados a partir de uma instância de processo. Cada evento possui um conjunto de atributos e são ordenados no tempo [van der Aalst 2016]. Um log é composto por atributos e outras propriedades adicionais tais como a identificação da instância (case), rastro (trace), eventos (events) e outros atributos que podem variar de acordo com o domínio (atributos dinâmicos). O histórico de eventos utilizado retrata os incidentes registrados pelo departamento de tecnologia da informação de uma empresa brasileira entre os anos de 2015 e 2016. Os incidentes são definidos por qualquer falha operacional de um serviço de TI, isto é, qualquer falha que envolva softwares, hardwares e quaisquer outros periféricos tais como servidores, impressoras e telefonia. Neste estudo de caso, considera-se a existência de SLA com tempo de resolução de problemas fixado em 720 minutos, i.e., cada instância de processo com o tempo decorrido desde o momento da sua abertura até o momento de fechamento maior que 720 minutos é considerada uma instância com uma situação de fracasso. Por meio dos fracassos evidenciados no log é possível mensurar o desacordo entre a realidade em que o modelo de processo foi idealizado com a realidade atual do negócio e prestação dos serviços.

O cenário considerado possui um total de 294628 eventos e 7259 instâncias de processo. O log de Eventos possui os seguintes atributos:

\section{Atributos da instância do processo:}

Case. identifica o incidente tratado.

OpenTime. registra o momento da abertura do incidente.

CloseTime. registra a data de encerramento do incidente.

Customer. identifica o cliente ou o responsável por abrir o incidente frente ao sistema.

Service. identifica se o incidente está relacionado a instalação e manutenção de softwares, hardwares ou manutenção da rede local.

\section{Atributos dos eventos do processo:}

EventId. identifica o evento em um nível transacional.

EventName. distingue os nomes dos eventos ou atividades do processo.

Article. identifica se houve trocas de mensagens entre os envolvidos do incidente, i.e., entre quem abriu o incidente com o técnico responsável por atender o incidente.

Priority. atributo numérico que pode variar de 1 à 5 .

TimeStamp. dia e a hora que o evento foi registrado pelo sistema; ordenação topológica da cadeia de eventos de uma instância de processo.

\subsection{Enriquecimento de Dados}

A preparação de dados consiste de três tarefas: (i) Calcular o indicador para cada instância do processo; (ii) momento de corte para a modelagem preditiva e (iii) enriquecimento do histórico de eventos com informações dos modelos de processos. O indicador é obtido por meio de duas etapas: (i) obter o tempo decorrido entre o primeiro evento da instância do processo e o evento da iteração do momento; e (ii) testar se tempo decorrido é maior que 720 minutos. Após a segunda etapa, atribui-se os devidos indicadores de fracasso para a instância do processo e também para o evento da iteração.

A Figura $2 \mathrm{a}$ apresenta um modelo de processo como exemplo, enquanto a Figura $2 \mathrm{~b}$ apresenta as instâncias deste processo. As instâncias possuem eventos que estão presentes em uma instância e não estão presentes em outras (eventos D e E) devido à ramificação do processo. No exemplo, 
Case 1 tem uma variante e Case 2 e Case 3 apresentam uma segunda variante. Para a abordagem preditiva, é necessário que o modelo seja construído de uma maneira antecipada ao evento que demarca o fracasso. Assim, sugere-se um momento de corte de eventos a, no mínimo, um evento de antecedência ao evento que possui o atributo de fracasso.

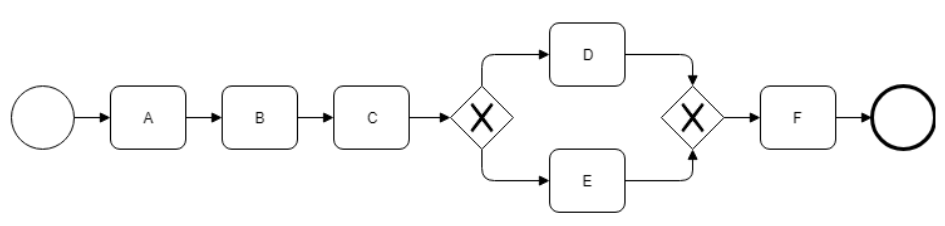

(a)

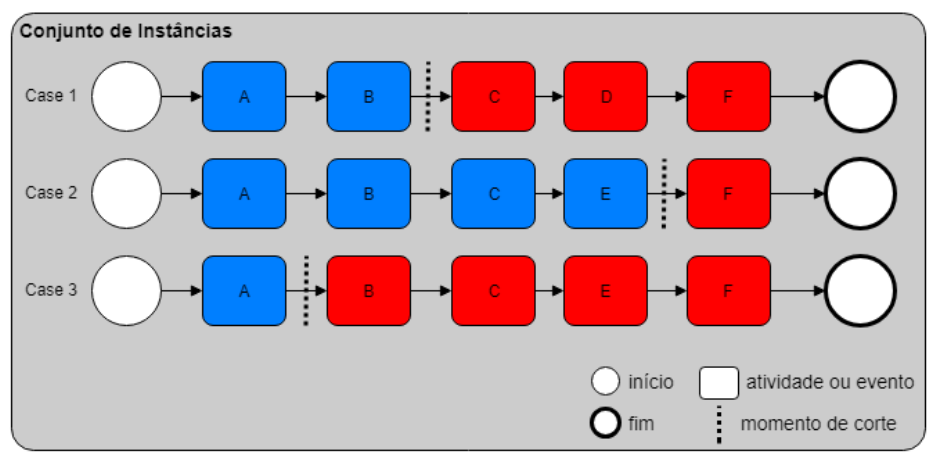

(b)

Fig. 2: Modelo de processo e suas instâncias com fracasso. (a) Modelo de processo idealizado por meio de seu fluxo operacional, (b) instâncias do processo que fracassaram em diferentes atividades.

Considerando a Figura 2b, para a primeira instância (Case 1), os eventos A e B antecedem um evento com atributo da situação de fracasso, e são os eventos considerados para a abordagem proposta. Os fracassos ocorrem em diferentes momentos do fluxo. O momento de corte é determinado por meio do evento que antecede o primeiro evento com o atributo identificador de fracasso (representados na cor vermelha). Após a identificação do momento de corte, removem-se todos os eventos que o precedem, pois os próximos eventos já não possuem relevância para a predição dado que a situação de fracasso já ocorreu. Em seguida, todas as instâncias têm o momento de corte parametrizado de acordo com o número mínimo de eventos das instâncias com indicador de fracasso. Desta maneira, equilibramos o número de eventos para todas as instâncias, independente do indicador. Assim, todas as instâncias são tratadas de maneira incompleta, isto é como se ainda estivessem em execução.

Feito isso, o log é enriquecido para identificar as variantes de processos que caracterizam padrões que entregam bons resultados. Nesta etapa, o algoritmo Fuzzy Miner [Günther and van der Aalst 2007] é utilizado para a descoberta dos modelos de processos após o momento de corte. Ao final, têm-se um $\log \mathcal{L}^{\prime}$ obtido a partir de $\mathcal{L}$. O log é enriquecido com os seguintes atributos:

Events. número de eventos que a instância de processo teve.

Variant. identificador da variante de processo.

Started. primeiro momento que um evento de uma determinada instância foi registrado.

Finished. último momento que um determinado evento de uma instância foi registrada.

Duration. tempo decorrido de uma determinada instância do processo.

\subsection{Event Encoding}

Esta etapa visa obter uma representação do histórico de eventos como um vetor de variáveis, também conhecida por Event Encode [Marquez-Chamorro et al. 2017]. Diferentes técnicas podem ser aplicadas no Event Encoding, por exemplo, (i) boolean encoding; (ii) frequency-based encoding e (iii) index-based encoding ${ }^{2}$.

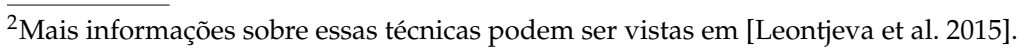


Para obter a representação final do $\log \mathcal{L}^{\prime}$ como vetor de variáveis, é preciso tratar os atributos que apresentam variações entre eventos de uma mesma instância. Para isso, o método proposto combina duas técnicas: boolean encoding, onde atribui-se uma relação de pertinência para cada variável que corrasponda a uma classe de eventos (ou atividades) do processo; e frequency encoding, onde soma-se cada classe de eventos que estabeleceram as relações de pertinência. Desta maneira, não se perde as informações dos eventos e por meio do frequency encoding é possível identificar se há mudanças de um mesmo atributo em tempos distintos de uma instância do processo.

\subsection{Pipeline de processamento}

A construção do modelo de predição se dá por meio de um fluxo de atividades, também chamado de pipeline de processamento, que produz como resultado final um modelo capaz de estimar o atributo referente ao fracasso de uma instância de processo. O pipeline de processamento proposto é apresentado na Figura $3 \mathrm{em}$ duas fases (a) Pipeline referente a fase de treinamento e (b) Pipeline referente a fase de predição, avaliação e teste.

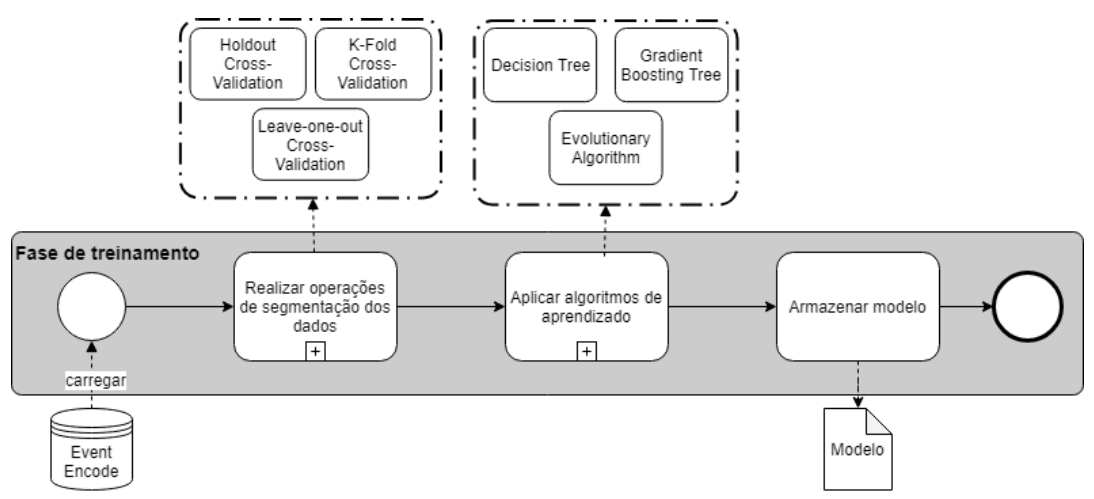

(a)

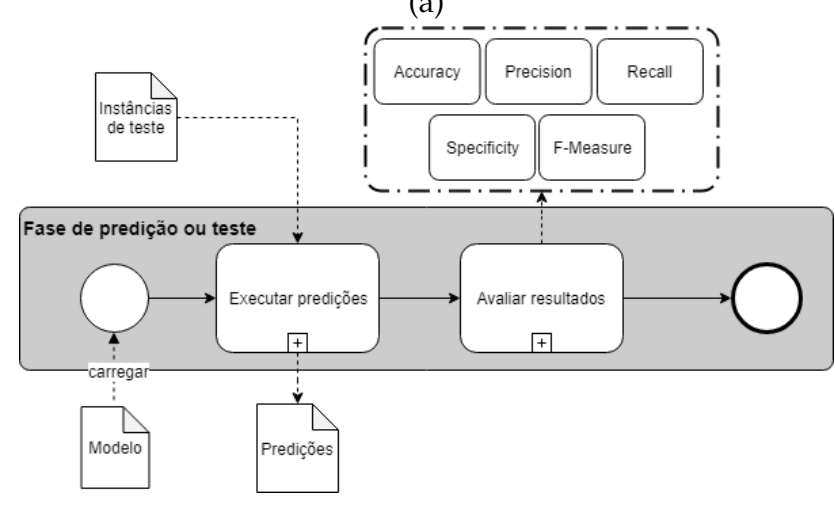

(b)

Fig. 3: Pipeline de construção do modelo de predição em duas fases. (a) Pipeline referente a fase de treinamento, (b) Pipeline referente a fase de predição, avaliação e teste.

Considerando a Figura 3a, o fluxo de atividades da fase de treinamento é dado por:

Realizar operações de segmentação dos dados. estratégia de separação do conjunto de dados entre instâncias de treinamento e teste; exemplos: Holdout Cross-Validation, K-Fold Cross-Validation e Leave-one-out Cross-Validation [Kohavi 1995]; 
Aplicar algoritmos de aprendizado. algoritmos de aprendizado de máquina que recebem como entrada o conjunto de instâncias de treinamento, obtidas por meio da atividade anterior; exemplos: Decision Tree, Gradient Boosting Tree e Evolutionary Algorithm;

Armazenar modelo. modelo treinado para avaliações futuras; dependendo da estratégia de segmentação adotada, diversos modelos são gerados.

Na segunda fase da construção do modelo de predição (Figura 3b), o fluxo é dado por:

Executar predições. as predições são realizadas no conjunto de dados de teste obtido por meio da estratégia de segmentação adotada produzindo os resultados, referentes à variável que indica o fracasso, que o modelo estima ser como assertivo;

Avaliar resultados. a avaliação é realizada comparando os resultados que o modelo estimou com os resultados reais das instâncias de teste; exemplos de métricas: Accuracy, Precision, Recall, Specificity.

\section{RESULTADOS E DISCUSSÃO}

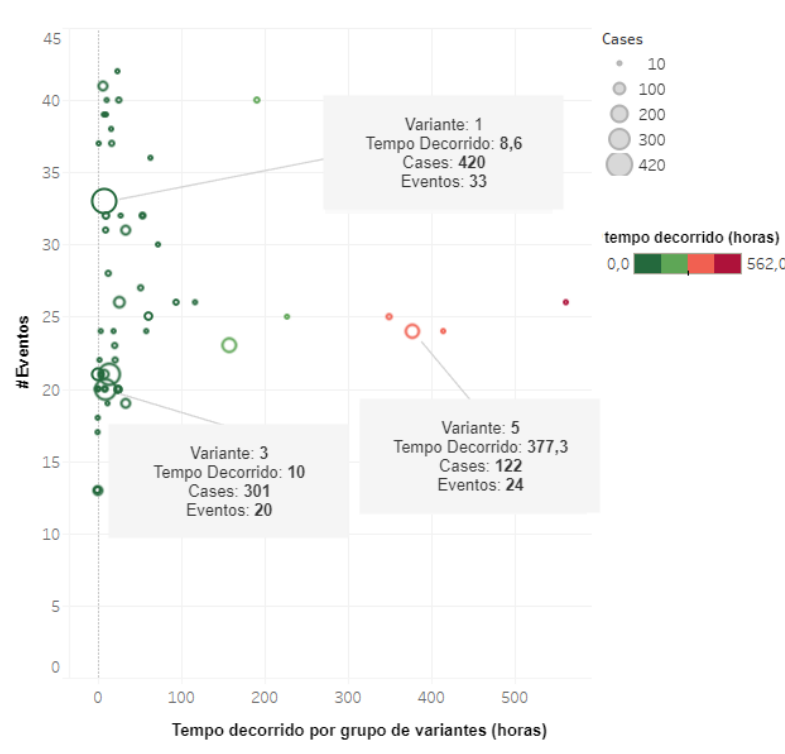

Fig. 4: Gráfico de dispersão utilizado como verificação da capacidade de discriminação do conjunto de dados por meio das variantes de processo. No eixo-x: total de eventos que uma variante de processo possui; eixo-y: Tempo médio decorrido.
A Figura 4 mostra um gráfico de dispersão que permite observar se algumas variantes do processo do estudo de caso fracassaram com mais frequência e, desta maneira, concluir quais podem ser mais suscetíveis ao fracasso. Destacam-se três variantes $(1,3$ e 5 ) em termos de volume de instâncias de processo onde as variantes 1 e 3 possuem o tempo médio decorrido inferior à 720 minutos, isto é, inferior à 12 horas. Por outro lado, a variante de processo identificada pelo número 5 é caracterizada por possuir um volume considerável de instâncias de processo (122 instâncias) que possuem tempo superior à 377 horas. Há muitas outras variantes de processo que poderiam ser desconsideradas do log por possuírem baixo volume de instâncias de processo e, sendo assim, de baixa significância. Desta maneira, pode-se notar que há variantes de processo que entregam bons resultados.

A etapa de corte resultou em variantes de processo cujo tamanho mínimo são 8 eventos. Isto implica que após o oitavo evento muitas instâncias fracassaram. Sendo assim, todas as instâncias do experimento foram delimitadas para o total de 8 eventos a partir da abertura do incidente. Após obter o vetor de variáveis dado pelo Event Encoding, foi realizado a segmentação do conjunto de dados. A forma de segmentação entre os conjuntos de treinamento e testes da predição se deu por meio da técnica $k$-fold cross-validation ${ }^{3}$. Na sequência, os seguintes algoritmos foram aplicados: Decision Tree (DT), Random Forest (RF) e Gradient Boosted Tree (GBT) ${ }^{4}$. Para avaliar a

\footnotetext{
${ }^{3} \mathrm{~A}$ técnica $k$-fold cross-validation consiste em segmentar em $k$ partições de tamanhos iguais. Uma das partes é reservada para o conjunto de teste e as outras $k-1$ partes são utilizadas na fase de treinamento. O particionamento de $k$ partições se dá aleatoriamente.

${ }^{4}$ Mais informações sobre esses algoritmos podem ser vistas em [Breiman 2017; Hastie et al. 2009].
} 
confiança dos resultados obtidos após a aplicação dos algoritmos, este artigo adota as seguintes medidas: Acurácia (Accuracy), Precisão (Precision), Sensibilidade ou Revocação (Recall), Especificidade (Specificity) e Medida F (F Measure) [Fawcett 2006; Witten et al. 2016].

Os resultados da Tabela I referem-se à apuração média para 10-fold cross-validation .

Considerando a Tabela I, o algoritmo GBT se destaca em três resultados se comparado a DT e RF. Esses resultados são: (i) accuracy, (ii) precision e (iii) Fmeasure. O algoritmo RF se destaca em três resultados se comparado com DT. Atribuímos a superioridade de GBT e RF se comparado a DT à técnica conhecida por Métodos de Ensemble. Os Métodos de Ensemble emergem a partir da hipótese de que a combinação de diferentes algoritmos de base podem melhorar a acurácia de um determinado problema [Hastie et al. 2009]. Essa hipótese é válida para o domínio explorado por este artigo. Ainda em relação aos resultados apresentados na Tabela I, atribuímos a superioridade de GBT se comparado a RF à técnica conhecida por Boosting. Técnicas de Boosting se encontram dentro dos Métodos de Ensemble e consistem da utilização de versões ponderadas do mesmo conjunto de treinamento para os algoritmos de base [Schapire and Freund 2012; Hastie et al. 2009].

\section{TRABALHOS RELACIONADOS}

Os trabalhos relacionados são classificados em: predição de eventos ou atividades de processos; predição de modelos de processos de negócio e predição de indicadores de desempenho de processos de negócio. Para cada um dos três existem duas abordagens possíveis: técnicas cientes de processos e técnicas não-cientes de processos. As técnicas cientes de processos caracterizam-se por considerar a estrutura do processo, isto é, a transição entre as atividades e os respectivos grafos direcionados são considerados por essas técnicas. Por outro lado, as técnicas não-cientes de processos são aplicadas em dados que não remetem uma estrutura encadeada de eventos isto é, não considera a transição entre as atividades do processo e os grafos direcionados dos mesmos. [Maggi et al. 2014], [Leontjeva et al. 2015] e [Márquez-Chamorro et al. 2017] são exemplos de técnicas não-cientes de processos e [Breuker et al. 2016] aplica uma técnica ciente de processos. [Maggi et al. 2014] utilizam o algoritmo Decision Tree para predição das violações das restrições de negócio em um estudo de caso no domínio de diagnósticos médicos e avaliam seus resultados por meio das métricas Accuracy, Precision, Recall, Specificity. [Leontjeva et al. 2015] mostram que o enriquecimento das formas de encoding com informações probabilísticas melhora em alguns casos a confiabilidade da predição. [Márquez-Chamorro et al. 2017] descobrem um conjunto de padrões adotando uma janela de eventos (Event Window) para aprimorar ou fornecer o apoio operacional em estratégias de mitigação de riscos. [Breuker et al. 2016] propõem uma abordagem baseada em técnicas de Mineração de Processos e Inferência Gramatical para predição de modelos de processos, isto é, de eventos de um processo.

\section{CONCLUSÕES E TRABALHOS FUTUROS}

No presente trabalho de pesquisa, apresentamos um estudo empírico que aplica diferentes técnicas da área de Mineração de Dados e Mineração de Processos de maneira complementar. Essa complementaridade se dá por meio da combinação dessas técnicas com o objetivo de preencher determinadas lacunas em alguns passos que a predição de uma variável ou atributo do processo necessita em termos de dependência. No estudo aqui exposto, essa variável é referenciada como um indicador de desempenho da instância do processo e indica se o processo possui o desempenho esperado em termos do 
alcance dos objetivos estratégicos e operacionais do processo. O Event Encoding considerado segue uma abordagem especial, a sumarização das atividades se dá por meio dos resultados do Fuzzy Miner onde não seria necessário aplicar a forma index-based encoding, uma vez que, o fluxo está representado diretamente nas informações de variantes de processo. As formas boolean encoding e frequency-based encoding são utilizados de maneira complementar nos atributos dinâmicos. Em relação à predição dos fracassos das instâncias do processo, foi verificado o potencial de três algoritmos para lidar com a falta de benchmarks internacionalmente aceitos. A técnica apresentada é não-ciente de processo e, a principal diferença da técnica deste artigo em relação aos trabalhos apresentados na Seção 4 está no uso das informações das variantes de processos pois nenhuma das abordagens avaliaram o que essas informações poderiam agregar em um contexto de predição.

Por fim, o pipeline de processamento mostrou-se bem flexível para a aplicação das diferentes técnicas e algoritmos. Concluímos que o trabalho alcançou bons resultados para a predição com o algoritmo GBT como destaque. Como trabalho futuro, pretende-se implementar o método aqui exposto como um plugin do Software ProM ${ }^{5}$ e aplicar o método apresentado em outros domínios e obter novos benchmarks representativos considerando também o uso das técnicas cientes de processos.

\section{REFERENCES}

Breiman, L. Classification and regression trees. Routledge, 2017.

Breuker, D., Matzner, M., Delfmann, P., and Becker, J. Comprehensible predictive models for business processes. MiS Quarterly 40 (4): 1009-1034, 2016.

del Río-Ortega, A., García, F., Resinas, M., Weber, E., Ruiz, F., and Ruiz-Cortés, A. pp. 193-209. In E. Dubois and K. Pohl (Eds.), Enriching Decision Making with Data-Based Thresholds of Process-Related KPIs. Springer International Publishing, Cham, pp. 193-209, 2017.

del Río-Ortega, A., Gutiérrez, A. M., Durán, A., Resinas, M., and Ruiz-Cortés, A. Modelling service level agreements for business process outsourcing services. In Advanced Information Systems Engineering, J. Zdravkovic, M. Kirikova, and P. Johannesson (Eds.). Springer International Publishing, Cham, pp. 485-500, 2015.

FAwcett, T. An introduction to roc analysis. Pattern recognition letters 27 (8): 861-874, 2006.

Günther, C. ANd van der Aalst, W. Fuzzy mining-adaptive process simplification based on multi-perspective metrics. Business Process Management, 2007.

Hastie, T., Tibshirani, R., and Friedman, J. The Elements of Statistical Learning. Bayesian Forecasting and Dynamic Models vol. 1, pp. 1-694, 2009.

Hompes, B. F. A., Maaradji, A., La Rosa, M., Dumas, M., Buijs, J. C. A. M., and van der Aalst, W. M. P. Discovering Causal Factors Explaining Business Process Performance Variation. In CAiSE. Vol. 7328. pp. 177-192, 2017.

Kohavi, R. A Study of Cross-Validation and Bootstrap for Accuracy Estimation and Model Selection, 1995.

Leontjeva, A., Conforti, R., Di Francescomarino, C., Dumas, M., and Maggi, F. M. Complex symbolic sequence encodings for predictive monitoring of business processes. In International Conference on Business Process Management. Springer, pp. 297-313, 2015.

Maggi, F. M., Di Francescomarino, C., Dumas, M., and Ghidini, C. pp. 457-472. In M. Jarke, J. Mylopoulos, C. Quix, C. Rolland, Y. Manolopoulos, H. Mouratidis, and J. Horkoff (Eds.), Predictive Monitoring of Business Processes. Springer International Publishing, Cham, pp. 457-472, 2014.

Marquez-Chamorro, A. E., Resinas, M., and Ruiz-Cortes, A. Predictive monitoring of business processes: a survey, 2017.

Márquez-Chamorro, A. E., Resinas, M., Ruiz-Cortés, A., and Toro, M. Run-time prediction of business process indicators using evolutionary decision rules. Expert Systems with Applications vol. 87, pp. 1-14, 2017.

Rudzajs, P. And Kirikova, M. Advanced Information Systems Engineering. In CAiSE 2018, J. Krogstie and H. A. Reijers (Eds.). Lecture Notes in Computer Science, vol. 10816. Springer International Publishing, 2018.

Schapire, R. E. ANd Freund, Y. Boosting: Foundations and algorithms. MIT press, 2012.

Van Der Aalst, W. M. Business process management: a comprehensive survey. ISRN Software Engineering, 2013.

van der Aalst, W. M. Process Mining: Data Science in Action. Springer-Verlag Berlin Heidelberg, 2016.

Weske, M., Marco Montali, Ingo Weber, and Jan vom Brocke. Business Process Management. In Business Process Management, M. Weske, M. Montali, I. Weber, and J. vom Brocke (Eds.). Lecture Notes in Computer Science, vol. 11080. Springer International Publishing, 2018.

Witten, I. H., Frank, E., Hall, M. A., And Pal, C. J. Data Mining: Practical machine learning tools and techniques. Morgan Kaufmann, 2016.

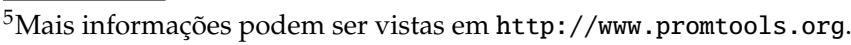

